\title{
Zubrod Performance Status 4
}

National Cancer Institute

\section{Source}

National Cancer Institute. Zubrod Performance Status 4. NCI Thesaurus. Code C17848.

Completely disabled. Cannot carry on any self-care. Totally confined to bed or chair (Eastern Cooperative Oncology Group) 